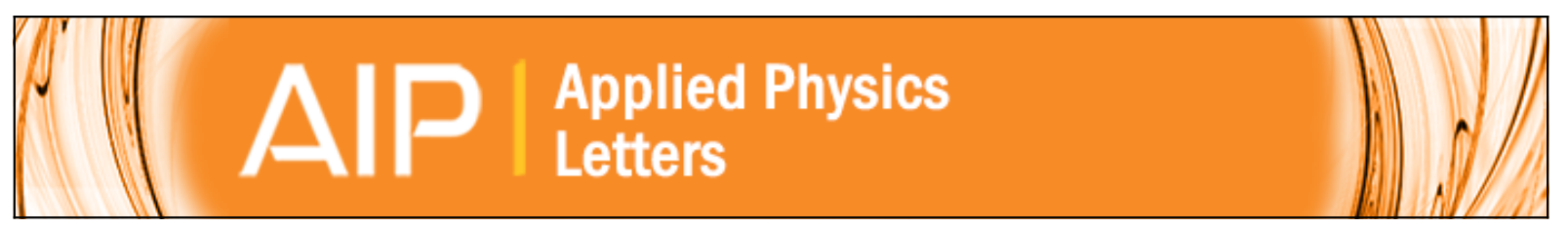

\title{
Compact extreme ultraviolet source for laboratory-based photoemission spectromicroscopy
}

Christoph Schmitz, Daniel Wilson, Denis Rudolf, Carsten Wiemann, Lukasz Plucinski, Sally Riess, Martin Schuck , Hilde Hardtdegen, Claus M. Schneider, F. Stefan Tautz, and Larissa Juschkin

Citation: Applied Physics Letters 108, 234101 (2016); doi: 10.1063/1.4953071

View online: http://dx.doi.org/10.1063/1.4953071

View Table of Contents: http://scitation.aip.org/content/aip/journal/apl/108/23?ver=pdfcov

Published by the AIP Publishing

\section{Articles you may be interested in}

Note: Thickness determination of freestanding ultra-thin foils using a table top laboratory extreme ultraviolet source

Rev. Sci. Instrum. 84, 056109 (2013); 10.1063/1.4807153

Plasma-oxidation of $\mathrm{Ge}(100)$ surfaces using dielectric barrier discharge investigated by metastable induced electron spectroscopy, ultraviolet photoelectron spectroscopy, and x-ray photoelectron spectroscopy

J. Appl. Phys. 110, 033302 (2011); 10.1063/1.3611416

Tunable ultrafast extreme ultraviolet source for time- and angle-resolved photoemission spectroscopy

Rev. Sci. Instrum. 81, 073108 (2010); 10.1063/1.3460267

Properties of $\mathrm{p}-\mathrm{n}$ heterojunction diode based on $\mathrm{Ge} 2 \mathrm{Sb} 2 \mathrm{Te} 5$ and its application for phase change random access memory

J. Appl. Phys. 105, 061627 (2009); 10.1063/1.3055417

Potential of discharge-based lithium plasma as an extreme ultraviolet source

Appl. Phys. Lett. 89, 031503 (2006); 10.1063/1.2227560

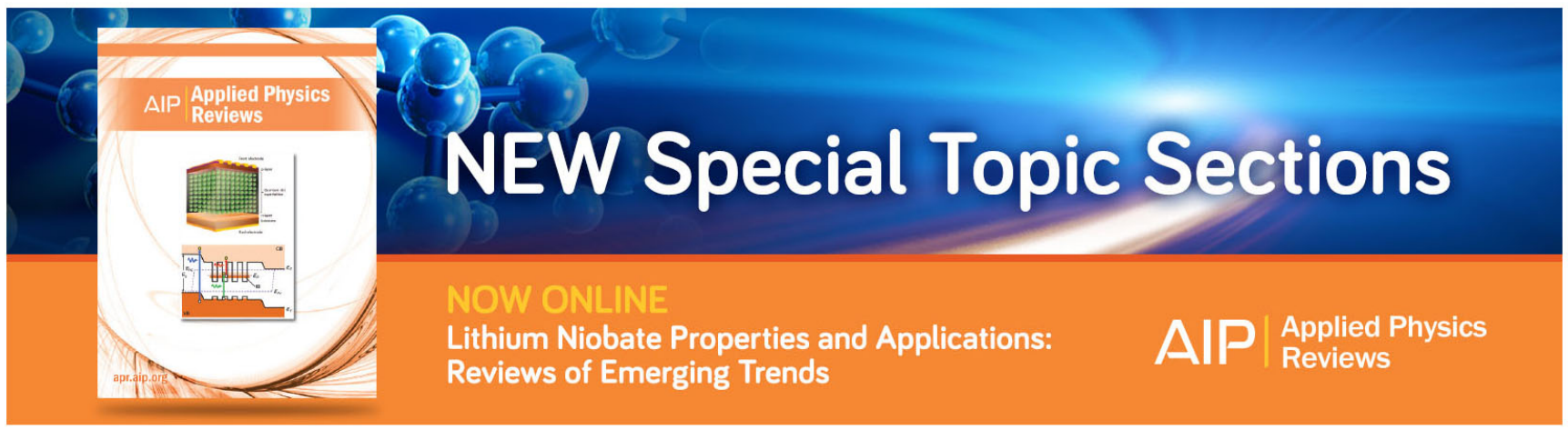




\title{
Compact extreme ultraviolet source for laboratory-based photoemission spectromicroscopy
}

\author{
Christoph Schmitz, ${ }^{1, a)}$ Daniel Wilson, ${ }^{1,2}$ Denis Rudolf, ${ }^{1,2}$ Carsten Wiemann, ${ }^{1}$ \\ Lukasz Plucinski, ${ }^{1}$ Sally Riess, ${ }^{1}$ Martin Schuck, ${ }^{1}$ Hilde Hardtdegen, ${ }^{1}$ Claus M. Schneider, ${ }^{1}$ \\ F. Stefan Tautz, ${ }^{1}$ and Larissa Juschkin ${ }^{1,2}$ \\ ${ }^{1}$ Peter Grünberg Institut, Forschungszentrum Jülich, JARA-FIT, 52425 Jülich, Germany \\ ${ }^{2}$ Experimental Physics of EUV, RWTH Aachen University, JARA-FIT, 52074 Aachen, Germany
}

(Received 31 December 2015; accepted 22 April 2016; published online 6 June 2016)

\begin{abstract}
We report on the combination of a state-of-the-art energy-filtering photoemission electron microscope with an intense yet compact laboratory-based gas discharge extreme ultraviolet (EUV) light source. Using a photon energy of $71.7 \mathrm{eV}$ from oxygen plasma $\left(\mathrm{O}^{5+}\right.$ spectral line), we demonstrate element-selective photoelectron imaging in real space and band structure mapping in reciprocal space. Additionally, the high surface sensitivity of the EUV light was used to study the surface oxidation on islands of the phase-change material $\mathrm{Ge}_{1} \mathrm{Sb}_{2} \mathrm{Te}_{4}$. The EUV light source allows the extension of spectromicroscopy, previously only feasible at synchrotron beamlines, to laboratory-based work. Published by AIP Publishing. [http://dx.doi.org/10.1063/1.4953071]
\end{abstract}

With the upsurge of functionalized microstructures in various academic disciplines, the spatially resolved characterization of the chemical and electronic properties on the sub-micron scale becomes progressively more important. If a photoemission electron microscope is combined with an energy analyzer, the imaging of energy-filtered electrons becomes possible (EF-PEEM). Such instruments are known as spectromicroscopes. To exploit the full potential of spectromicroscopy, an intense, monochromatic yet tunable light source of defined polarization in the extreme ultraviolet (EUV) and soft X-ray spectral range is required. Therefore, spectromicroscopy is commonly carried out at undulator beamlines of 3rd generation synchrotron sources. ${ }^{1}$ However, this limits its use to a small scientific community. If an intense laboratory-based light source tunable in the suitable spectral range was available, the application potential of spectromicroscopy could be vastly enhanced, allowing for both electronic structure (valence band spectroscopy) and chemical composition (core level spectroscopy) imaging of many samples on a routine basis. In this context, EUV radiation plays an important role, as photoemission with EUV light offers several advantages. First, the photoionization cross section for many elements is maximized in the EUV ensuring high photoemission signals. ${ }^{2,3}$ Second, the electron inelastic mean free path (IMFP) has a minimum of around $70 \mathrm{eV}$ of electron kinetic energy with an average value below $1 \mathrm{~nm}$ maximizing the surface sensitivity. ${ }^{4,5}$ Unfortunately, however, the currently available standard laboratory-based light sources for photoemission ${ }^{6}$ do not cover the EUV spectral gap between $40.8 \mathrm{eV}$ (UPS) and $1.235 \mathrm{keV}$ (X-ray photoelectron spectroscopy, XPS). This calls for the development of a suitable EUV light source.

In this letter, we report on proof-of-concept experiments combining an intense, yet compact gas discharge plasma EUV light source and a state-of-the-art PEEM (based on a

\footnotetext{
a) Author to whom correspondence should be addressed. Electronic mail: c.schmitz@fz-juelich.de
}

Focus NanoESCA ${ }^{7-11}$ ). The EUV source emits in the spectral range between vacuum ultraviolet $(20 \mathrm{eV})$ and soft $\mathrm{X}$-rays $(600 \mathrm{eV})$, thus covering the lower part of the spectral gap and allowing for electronic and chemical analyses in a single experiment. To demonstrate the feasibility of the approach, we show examples of real space spectromicroscopy and reciprocal space band imaging at a photon energy of $h \nu=71.7 \mathrm{eV}$.

Initially designed for EUV lithography, ${ }^{12}$ the EUV source was already applied in EUV microscopy ${ }^{13,14}$ and spectroscopy. ${ }^{15}$ Using a high current discharge to ignite a gas plasma, the source generates a spectrum of narrowbandwidth $\left(\Delta E / E=10^{-3}-10^{-5}\right)$ spectral lines with highest intensity between $20 \mathrm{eV}$ and $600 \mathrm{eV}$ photon energy emitted by multiple ionized atoms, e.g., $\mathrm{O}^{2+}-\mathrm{O}^{6+}$, etc. The abundance and the intensity of the observed spectral linescorresponding to the emitted photon energies-strongly depends on the plasma temperature, plasma density, and gas composition. A typical spectrum of an oxygen plasma, which is used in this study, is shown in the inset of Fig. 1. The EUV source operates in a pulsed charge-discharge mode with a tunable repetition frequency in the range of $1.5-2.5 \mathrm{kHz}$, whereby each discharge generates an EUV pulse with an average pulse length of several tens of nanoseconds. Due to the high brightness of the source, the setup is expected to fulfill the requirements for live image acquisition in (EF-) PEEM. The challenge in combining this light source with a photoemission setup mainly lies in the construction of the transfer optics that is necessary to monochromatize and focus the EUV light onto the sample. To minimize the EUV intensity losses caused by absorption, the full setup is operated under vacuum, and only reflecting optics are used. Additionally, to maintain the required UHV conditions in the analyzer $\left(10^{-9}\right.$ mbar) while having plasma operation conditions $\left(10^{-1}\right.$ mbar $)$ in the discharge region of the source, a differential pumping scheme and a thin, almost EUV transparent $\mathrm{Al}(150 \mathrm{~nm}) / \mathrm{C}(27 \mathrm{~nm})$ foil are used for vacuum separation. Since the emitted EUV spectrum consists of 

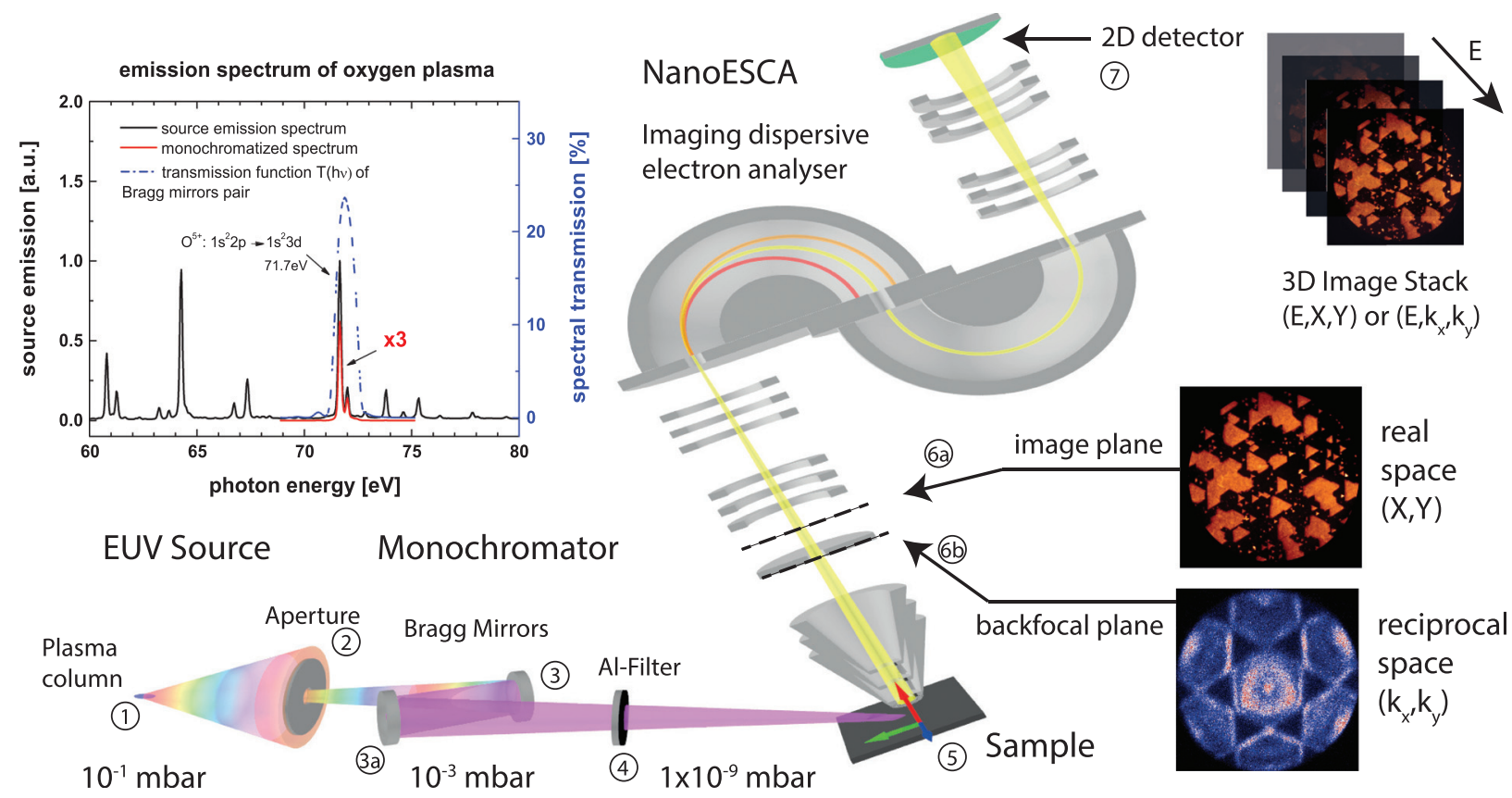

FIG. 1. Experimental setup: A discharge plasma within the EUV source generates a multi-wavelength EUV pulse (1). The radiation is collimated by a small aperture (2) and monochromatized (3) by a pair of narrow bandwidth Bragg mirrors (FWHM $=1.0 \mathrm{eV}$ ). The second mirror (3a) additionally focuses the monochromatized light onto the sample. An EUV-transparent Al filter of $150 \mathrm{~nm}$ thickness (4) is used for vacuum differentiation of the source and the UHV PEEM vacuum vessels. Photo-emitted electrons from the sample surface (5) are imaged by the EF-PEEM onto a 2D detector (7) behind two hemispherical analyzers. The microscope has two modes of operation: In real space mode (6a), the lateral intensity distribution $\mathrm{I}(x, y, E)$ of photoelectrons for different kinetic energies $E$ is projected. In reciprocal space mode (6b), the energy-dependent angular distribution of the electrons is measured and momentum maps I $\left(k_{x}, k_{y}, E\right)$ can be derived. Inset: Measured emission spectrum of oxygen plasma before monochromatization (black) and calculated spectrum behind the Bragg mirror pair (red). The blue line shows the spectral transmission of the Bragg mirror pair measured at the synchrotron. ${ }^{16}$

many isolated emission lines (Fig. 1, inset), each of which has a sufficiently small intrinsic linewidth $(\Delta E<10 \mathrm{meV}$ due to Doppler broadening in the case of oxygen ions), no widerange high-resolution monochromator is required. Instead, a set of Al/Mo-based Bragg mirrors consisting of a flat and a concave mirror ( $1 \mathrm{~m}$ radius) is used as narrow spectral bandwidth filter (FWHM $=1.0 \mathrm{eV}$ [Ref. 16]). The Bragg mirrors are optimized to select and focus only the spectral lines emitted by Li-like $\mathrm{O}^{5+}$ ions around $h \nu=71.7 \mathrm{eV}$ photon energy $\left(1 \mathrm{~s}^{2} 2 \mathrm{p}-1 \mathrm{~s}^{2} 3 \mathrm{~d}\right.$ doublet transition) onto the sample. The final spot size on the sample is $3.0 \times 1.7 \mathrm{~mm}^{2}$, and the total photon flux after spectral discrimination of $71.7 \mathrm{eV}$ photon energy was measured by a calibrated photodiode to be approximately $2 \times 10^{11}$ photons/s with a relative spectral resolution of about 1200. The photon flux and spectral resolution have the same order of magnitude as, e.g., the $\mathrm{BaD}$ ElPh vacuum ultraviolet photoemission beamline at Elettra. ${ }^{17}$ The performance of the setup using EUV excitation was tested by determining the spatial resolution with the help of a calibration sample. The calibration sample exhibits checkerboard structures consisting of neighboring Au and Si squares having a size of $1 \times 1 \mu \mathrm{m}^{2}$. PEEM images have been acquired with the EUV source $(71.7 \mathrm{eV})$, and, for reference, with a conventional $\mathrm{Hg}$ lamp $(5.2 \mathrm{eV}$ ) (see Fig. 2). The lens settings and the contrast aperture size have been kept the same for both measurements. The spatial resolution in the indicated region [green line in Fig. 2(a)] was determined by fitting a convolution of a Gaussian function and a step function to the measured line profile and extracting the full width at half maximum. For both photon energies, a sub-micron resolution was achieved. Specifically, the resolution amounts to $348.2 \pm 110 \mathrm{~nm}$
$(71.7 \mathrm{eV})$ and $151.3 \pm 9 \mathrm{~nm}(5.2 \mathrm{eV})$, respectively. The resolution derived for $\mathrm{Hg}$ excitation is in agreement with the instrumental resolution of $50-150 \mathrm{~nm}$ and the sample feature accuracy of $<200 \mathrm{~nm}$. The loss of spatial resolution at $71.7 \mathrm{eV}$ as compared to $5.2 \mathrm{eV}$ illumination may be attributed to (a) a reduced contrast for this specific photon energy and material combination, (b) chromatic aberrations due to a larger spread of electron kinetic energies, and (c) to the onset of space and surface mirror charges; for pulsed light sources, they are commonly observed to reduce the spatial resolution. ${ }^{18}$ All three effects have been observed in the course of our experiments. Further experiments are required to specifically quantify the origin and the impact of space charge effects. Next, we demonstrate the capability of the EUV source to perform chemically resolved spectromicroscopy based on core-level photoemission. To this end, we turned to a chemically (a)

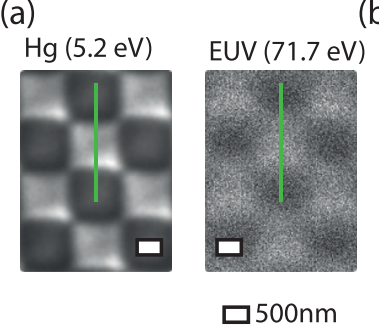

(b)

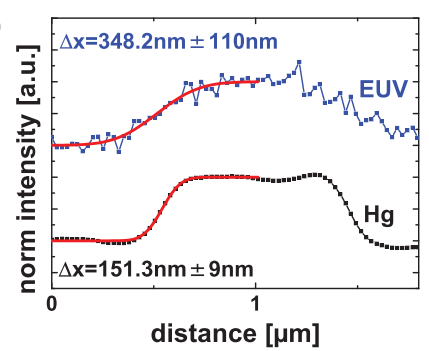

FIG. 2. PEEM images of a structured calibration sample with $1 \mu \mathrm{m} \times 1 \mu \mathrm{m}$ $\mathrm{Au}$ and $\mathrm{Si}$ squares at $5.2 \mathrm{eV}$ (left) and $71.7 \mathrm{eV}$ (right) photon energy. Both images show an average of 80 images at $10 \mathrm{~s}$ of integration time per image. The integrated intensity along the green lines together with fits of convolved Gaussian and step functions (red lines) are shown in (b). The spatial resolution is denoted by $\Delta x$. 
complex and microscopically structured, ternary material system. Specifically, we investigated crystalline hexagonal Ge$\mathrm{Sb}-\mathrm{Te}$ (GST, 1:2:4) islands epitaxially grown on $\mathrm{Si}(111)^{19}$ by metal organic vapor phase epitaxy (MOVPE). A particular focus of these experiments was on surface oxidation. GST is a prototype material for resistive switching based on phase change processes and it is well known from optical data storage in Compact Disc ReWriteable (CD-RW) and Digital Versatile Disc ReWriteable (DVD-RW) ${ }^{20}$ Prior to our photoemission studies, the ex-situ prepared sample was carefully cleaned by mild sputtering (Ar ions, $500 \mathrm{eV}$ ) and annealing at $250{ }^{\circ} \mathrm{C}$. Due to the high surface sensitivity of the method, this preparation step is mandatory. Fig. 3 shows EF-PEEM images of a GST sample recorded at $h \nu=5.2 \mathrm{eV}$ (a) and $h \nu=71.7 \mathrm{eV}$ (b). In both images, triangular-shaped islands ranging in size from several hundreds of nanometers up to $50 \mu \mathrm{m}$ and more are visible. The contrast in Fig. 3(a) arises from a difference in work function $\Phi$ between GST and Si. The bright regions correspond to GST, whereas the dark regions are the bare Si substrate. Orientation, alignment, and shape of the GST islands confirm the expected epitaxial growth on $\mathrm{Si}(111)$. Fig. 3(b) displays the spatially resolved tellurium concentration based on spectromicroscopy images acquired over the energy range of the spin-orbit split Te $4 \mathrm{~d}$ core level. In this case, the contrast is of chemical origin and stems from the presence or absence of Te atoms in the specific regions. Orange areas correspond to high Te concentrations and blue areas to low or zero Te concentrations. The Te intensity distribution clearly follows the shape of the GST islands as observed with the Hg lamp. Similar intensity distributions were acquired for $\mathrm{Sb}$ and $\mathrm{Ge}$ and their corresponding oxides
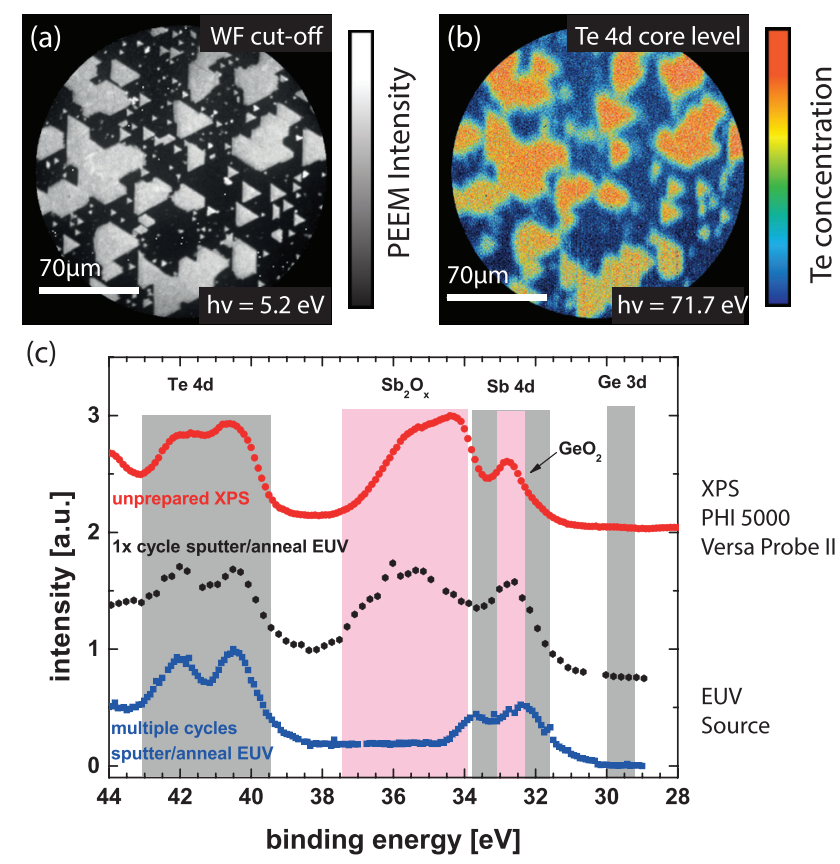

FIG. 3. EF-PEEM image of GST islands recorded at $\mathrm{h} \nu=5.2 \mathrm{eV}$ (a) and at $\mathrm{h} \nu=71.7 \mathrm{eV}$ (b) photon energy. Image (a) was acquired at the work function (WF) cut-off. Image (b) consists of integrated images around the $\mathrm{Te} 4 \mathrm{~d}$ core level binding energies $(39 \mathrm{eV}-41 \mathrm{eV})$. The image is slightly shifted with respect to (a) and shows space charge induced image deterioration. In panel (c), a binding energy spectrum of gently sputtered GST islands acquired with the EUV source (black) is compared to XPS reference data of a pristine sample (red). The oxide components of $\mathrm{Ge}$ and $\mathrm{Sb}$ are clearly visible. Additional preparation cycles lead to a purely metallic $\mathrm{Sb} / \mathrm{Te}$ termination (blue). (not shown). As all core-level distribution maps resemble the same intensity profile on the $\mu \mathrm{m}$ scale without variations between different islands, we assume the chemical composition of the islands to be homogeneous and independent of the island's size. A photoemission spectrum including only the signal from GST islands was extracted from the 3D image stack and is presented in Fig. 3(c) (black data points). We note that the acquisition time for each data point in the spectrum is only $4 \mathrm{~s}$. Still, the spin-orbit splitting of the Te $4 \mathrm{~d}$ core level can be clearly identified. ${ }^{21}$ In addition, we can identify the energyshifted $\mathrm{Sb} 4 \mathrm{~d}$ peak of $\mathrm{Sb}_{2} \mathrm{O}_{x}$ as a fingerprint of the oxidized surface of ex-situ prepared GST. Very likely, the Ge $3 \mathrm{~d}$ peak of $\mathrm{GeO}_{2}$ at about $33 \mathrm{eV}$ is also present in the spectrum, but obscured by the $\mathrm{Sb} 4 \mathrm{~d}$ peak. The observed oxide components confirm the anticipated surface oxidation. To compare our data with a well-established method, we performed XPS with Al $\mathrm{K}_{\alpha}$ radiation on the same sample in a standard XPS instrument (PHI 5000 VersaProbe II). Despite the different inelastic mean free paths $\left(\lambda_{\mathrm{IMFP}}<1 \mathrm{~nm}\right.$ (Ref. 5) at $h \nu=71.7 \mathrm{eV}$ compared to a few $\mathrm{nm}$ at $h \nu=1486.6 \mathrm{eV}$ ), the EUV (black) and X-ray photoemission (red) spectra are very similar (Fig. 3(c)). Application of additional cycles of sputter/annealing, however, completely removed the oxide layer in favor of a metallic $\mathrm{Te} /$ $\mathrm{Sb}$ termination (blue line in Fig. 3(c)).

Finally, to demonstrate the capability of the setup to perform angle-resolved photoemission spectroscopy (ARPES) and band structure mapping, the NanoESCA is used in its reciprocal ("k-space") mode to image the energy-dependent angular distribution of photoelectrons emitted from a $\mathrm{Au}$ (111) single crystal surface. The crystal was cleaned by multiple cycles of $\mathrm{Ar}$ ion sputtering and subsequent annealing to $500^{\circ} \mathrm{C}$. For the experiment, the energy resolution of the analyzer was set to $\Delta E=400 \mathrm{meV}$ in order to achieve the best compromise between transmission, energy resolution, and acquisition time. Here, the acquisition time ranges from 1 min per energy step for the high-intensity Au $5 \mathrm{~d}$ states ( $1-8 \mathrm{eV}$ binding energy) in the standard mode up to $10 \mathrm{~min}$ per image when approaching the Fermi level $E_{\mathrm{F}}$, where a special, single electron event counting mode ${ }^{10}$ was used. The calibration of the reciprocal field of view was performed by fitting a parabola to the secondary electron emission cone as described by Escher et al. ${ }^{9}$ At $71.7 \mathrm{eV}$ photon energy, the maximum observable field of view (FoV) is approximately $5.6 \AA^{-1}$. Within this FoV, the first as well as parts of the neighboring surface Brillouin zones can be imaged simultaneously (Fig. 4(a)). The symmetry of the (111) surface with indicated high symmetry points $\bar{\Gamma}, \bar{M}$ and $\bar{K}$ is clearly visible. In addition, the Shockley surface state (SS) ${ }^{22}$ located around $\bar{\Gamma}$ (starting $500 \mathrm{meV}$ below the Fermi edge, see inset) can be identified in Fig. 4(b) as a central bright spot. The measured k-radius of the state at $E_{\mathrm{F}}$ is around $0.13 \AA^{-1}$. Valence band photoemission spectra of the $A u$ (111) surface were extracted by integrating over the full acquired $\mathrm{k}$-space region $(\approx 2.5 \mathrm{BZ})$ at different binding energies. The resulting spectrum is displayed in Fig. 4(e). The observed double peak structure at $3 \mathrm{eV}$ and $6 \mathrm{eV}$ binding energy arises from the two Au $5 \mathrm{~d}$ bands, while the Fermi edge is mainly defined by the $\mathrm{Au} 6 \mathrm{~s}$ state. For a qualitative interpretation of the acquired k-maps, ab-initio band structure calculations (GGA-PBE) of bulk Au (111) including spin-orbit coupling 

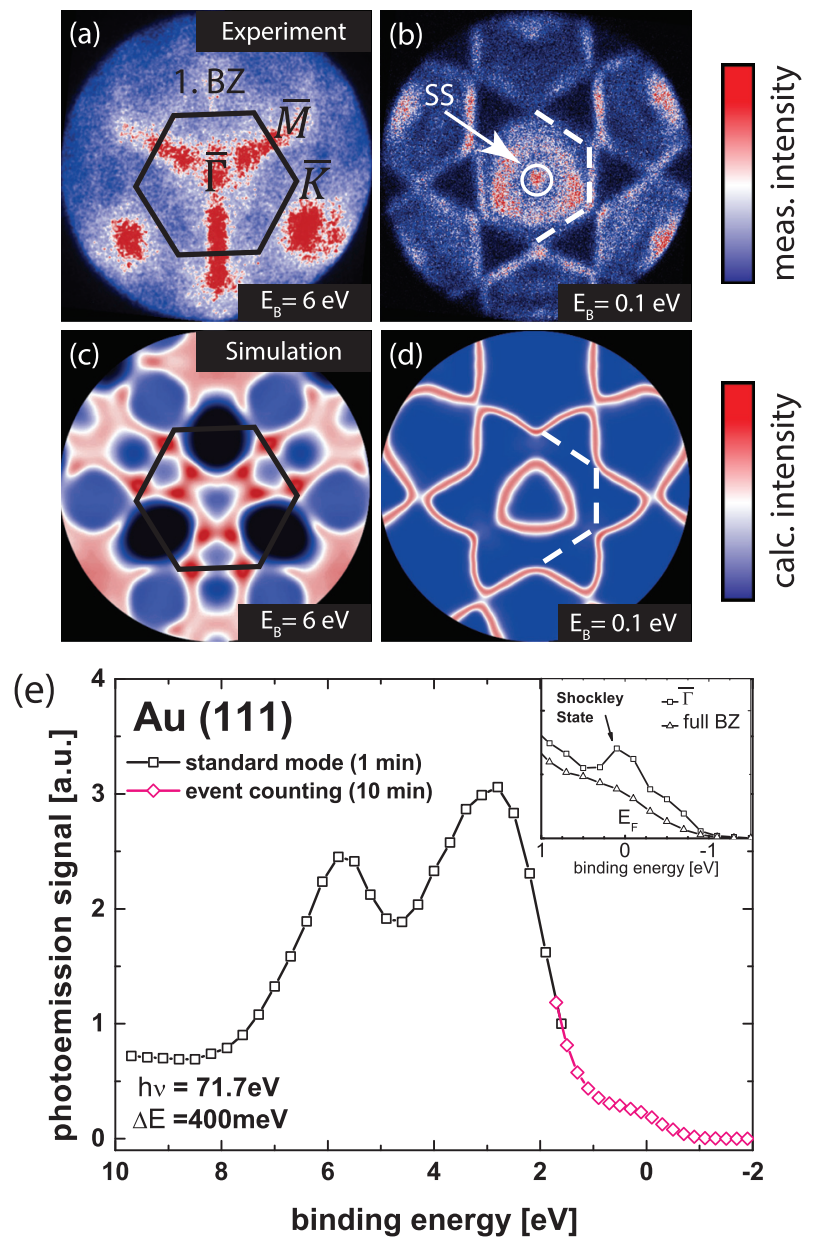

FIG. 4. Experimental k-space maps of Au 5d band (a) and Au(111) Fermi surface (b) showing a range of $5.6 \AA^{-1}$ in reciprocal space. The high symmetry points $\bar{\Gamma}, \bar{M}$ and $\bar{K}$ of the hexagonal surface Brillouin zone are marked within (a). Corresponding band structure calculations (WIEN2K) are shown in (c) and (d) for a qualitative comparison. The spectrum (e) was derived from integrated $\mathrm{k}$-space images recorded at different binding energies in the standard (black squares) as well as in the event counting mode ${ }^{10}$ (red diamonds) Inset: magnified view of the Fermi edge integrated over a k-space region around $\bar{\Gamma}$ only and integrated over the full Brillouin zone (BZ).

were performed using WIEN2K ${ }^{23}$ (Figs. 4(c) and 4(d)). The simulated raw data was artificially broadened by $400 \mathrm{meV}$ to match the energy resolution of the experiment. The main features and symmetries of the experiment can be reproduced by the simulation with a good agreement. However, it must be mentioned that some additional features, such as the additional hexagon located in the center of the Fermi surface (dashed line Fig. 4(b)), cannot be explained by those basic simulations. We attribute this feature to a second final state transition.

To conclude, we presented highly surface-sensitive spectromicroscopy with a laboratory-based gas-discharge EUV light source and a state-of-the-art energy filtered PEEM. Both real and reciprocal space imaging were demonstrated showing that EUV radiation with $h \nu=71.7 \mathrm{eV}$ from this source can be used to investigate the electronic valence structure as well as the chemical composition of a given sample in a single experiment. To put our results into perspective, only a few photoemission experiments (ARPES and PEEM) employing a laboratory-based EUV light source beyond He I (21.2 eV) and He II $(40.8 \mathrm{eV})$ have been reported so far, mostly using laser-generated high harmonics $(\mathrm{HHG})^{24-26}$ and laserproduced plasma light sources. $^{27-29}$ In future experiments, we plan to use a collector in combination with a grating monochromator to provide easy access to the different photon energies emitted by the source allowing for 3D molecular orbital tomography or depth-probing in XPS, and to further improve the energy resolution. Ultimately, the energy resolution of the source will be limited by Doppler broadening (about $10 \mathrm{meV}$ ) of the dominant spectral lines.

We acknowledge financial support from a JARA-FIT Seed Fund Project funded through the German Excellence Initiative. L.J. acknowledges financial support from the Helmholtz Association for a Helmholtz Professorship as a part of the Initiative and Networking Fund. We also thank B. Küpper and A. Bremen for their technical assistance throughout the project as well as A. Besmehn for XPS measurements. S.R., M.S., and H.H. as well as C.S., C.W., and C.M.S. acknowledge funding within the Collaborative Research Center 917 "Nanoswitches" by the Deutsche Forschungsgemeinschaft (DFG).

${ }^{1}$ C. Wiemann, M. Patt, I. P. Krug, N. B. Weber, M. Escher, M. Merkel, and C. M. Schneider, J. Surf. Sci. Nanotechnol. 9, 395 (2011).

${ }^{2}$ J. J. Yeh, Atomic Calculation of Photoionization Cross-sections and Asymmetry Parameters (Gordon \& Breach Science, 1993).

${ }^{3}$ J. J. Yeh and I. Lindau, Atomic Data and Nuclear Data Tables (Elsevier Science, 1985), Vol. 32, pp. 1-155.

${ }^{4}$ C. J. Powell and A. Jablonski, NIST Electron Inelastic-Mean-Free-Path Database, Version 1.2, National Institute of Standards and Technology, Gaithersburg, MD, 2010.

${ }^{5}$ M. P. Seah and W. A. Dench, Surf. Interface Anal. 1, 2 (1979).

${ }^{6}$ UPS: Ar: $11.6 \mathrm{eV}, \mathrm{Kr}: 10 \mathrm{eV}$, Ne: $16.848 \mathrm{eV}$ and $16.671 \mathrm{eV}$, He I: 21.22 eV, He II: $40.8 \mathrm{eV}, \mathrm{Hg}$ : $4.8 \mathrm{eV}$ and XPS: $\mathrm{Mg} \mathrm{K}_{\alpha}$ : $1253.5 \mathrm{eV}, \mathrm{Al} \mathrm{K}_{\alpha}$ : $1486.5 \mathrm{eV}$

${ }^{7}$ M. Escher, N. Weber, M. Merkel, C. Ziethen, P. Bernhard, G. Schönhense, S. Schmidt, F. Forster, F. Reinert, B. Krömker, and D. Funnemann, J. Phys.: Condens. Matter 17, 1329 (2005).

${ }^{8}$ N. Weber, M. Escher, M. Merkel, A. Oelsner, and G. Schönhense, J. Phys.: Conf. Ser. 100, 72031 (2008).

${ }^{9}$ M. Escher, K. Winkler, O. Renault, and N. Barrett, J. Electron Spectrosc. Related Phenom. 178-179, 303 (2010).

${ }^{10}$ M. Patt, C. Wiemann, N. Weber, M. Escher, A. Gloskovskii, W. Drube, M. Merkel, and C. M. Schneider, Rev. Sci. Instrum. 85, 113704 (2014).

${ }^{11}$ C. Wiemann, M. Patt, S. Cramm, M. Escher, M. Merkel, A. Gloskovskii, S. Thiess, W. Drube, and C. M. Schneider, Appl. Phys. Lett. 100, 223106 (2012).

${ }^{12}$ S. Danylyuk, H. Kim, S. Brose, C. Dittberner, P. Loosen, T. Taubner, K. Bergmann, and L. Juschkin, J. Vac. Sci. Technol. B 31, 21602 (2013).

${ }^{13}$ M. Benk, K. Bergmann, D. Schäfer, and T. Wilhein, Opt. Lett. 33, 2359 (2008).

${ }^{14}$ L. Juschkin, R. Freiberger, and K. Bergmann, J. Phys.: Conf. Ser. 186, 12030 (2009).

${ }^{15}$ D. Wilson, D. Rudolf, C. Weier, R. Adam, G. Winkler, R. Frömter, S. Danylyuk, K. Bergmann, D. Grützmacher, C. M. Schneider, and L. Juschkin, Rev. Sci. Instrum. 85, 103110 (2014).

${ }^{16}$ The Bragg mirrors were fabricated by optiX fab $\mathrm{GmbH}$. The reflectivity was measured at the PTB EUV reflectometry beamline in Berlin.

${ }^{17}$ L. Petaccia, P. Vilmercati, S. Gorovikov, M. Barnaba, A. Bianco, D. Cocco, C. Masciovecchio, and A. Goldoni, Nucl. Instrum. Methods Phys. Res. 606, 780 (2009).

${ }^{18}$ A. Locatelli, T. O. Menteş, M. Á. Niño, and E. Bauer, Ultramicroscopy 111, 1447 (2011).

${ }^{19}$ M. Schuck, S. Rieß, M. Schreiber, G. Mussler, D. Grützmacher, and H. Hardtdegen, J. Cryst. Growth 420, 37 (2015).

${ }^{20} \mathrm{M}$. Wuttig and N. Yamada, Nat. Mater. 6, 824 (2007).

${ }^{21}$ NIST X-ray Photoelectron Spectroscopy Database, Version 4.1 (National Institute of Standards and Technology, Gaithersburg, 2012); http://srdata.nist.gov/xps. 
${ }^{22}$ M. Hoesch, M. Muntwiler, V. N. Petrov, M. Hengsberger, L. Patthey, M. Shi, M. Falub, T. Greber, and J. Osterwalder, Phys. Rev. B 69, 241401 (2004).

${ }^{23}$ P. Blaha, K. Schwarz, G. Madsen, D. Kvasnicka, and J. Luitz, WIEN2K: An Augmented Plane Wave + Local Orbitals Program for Calculating Crystal Properties (Karlheinz Schwarz, Technische Universität Wien, Austria, 2001).

${ }^{24}$ S. Eich, A. Stange, A. V. Carr, J. Urbancic, T. Popmintchev, M. Wiesenmayer, K. Jansen, A. Ruffing, S. Jakobs, T. Rohwer, S. Hellmann, C. Chen, P. Matyba, L. Kipp, K. Rossnagel, M. Bauer, M. M. Murnane, H. C. Kapteyn, S. Mathias, and M. Aeschlimann, J. Electron. Spectrosc. Relat. Phenom. 195, 231 (2014).
${ }^{25}$ A. Mikkelsen, J. Schwenke, T. Fordell, G. Luo, K. Klünder, E. Hilner, N. Anttu, A. A. Zakharov, E. Lundgren, J. Mauritsson, J. N. Andersen, H. Q. Xu, and A. L'Huillier, Rev. Sci. Instrum. 80, 123703 (2009).

${ }^{26}$ S. H. Chew, F. Süßmann, C. Späth, A. Wirth, J. Schmidt, S. Zherebtsov, A. Guggenmos, A. Oelsner, N. Weber, J. Kapaldo, A. Gliserin, M. I. Stockman, M. F. Kling, and U. Kleineberg, Appl. Phys. Lett. 100, 51904 (2012).

${ }^{27}$ A. J. Arko, J. J. Joyce, and L. Morales, J. Alloys Compd. 286, 14 (1999).

${ }^{28}$ G. Schriever, S. Mager, A. Naweed, A. Engel, K. Bergmann, and R. Lebert, Appl. Opt. 37, 1243 (1998).

${ }^{29}$ H. Kondo, T. Tomie, and H. Shimizu, Appl. Phys. Lett. 72, 2668 (1998). 